\title{
Geometric Methods to Investigate Prolongation Structures for Differential Systems with Applications to Integrable Systems
}

\author{
Paul Bracken \\ Department of Mathematics, University of Texas, Edinburg, TX 78541-2999, USA \\ Correspondence should be addressed to Paul Bracken; bracken@utpa.edu
}

Received 8 January 2013; Accepted 13 February 2013

Academic Editor: Aloys Krieg

Copyright ( 2013 Paul Bracken. This is an open access article distributed under the Creative Commons Attribution License, which permits unrestricted use, distribution, and reproduction in any medium, provided the original work is properly cited.

\begin{abstract}
A type of prolongation structure for several general systems is discussed. They are based on a set of one forms for which the underlying structure group of the integrability condition corresponds to the Lie algebra of $S L(2, \mathbb{R}), O(3)$, and $S U(3)$. Each will be considered in turn and the latter two systems represent larger $3 \times 3$ cases. This geometric approach is applied to all of the three of these systems to obtain prolongation structures explicitly. In both $3 \times 3$ cases, the prolongation structure is reduced to the situation of three smaller $2 \times 2$ problems.
\end{abstract}

\section{Introduction}

Geometric approaches have been found useful in producing a great variety of results for nonlinear partial differential equations [1]. A specific geometric approach discussed here has been found to produce a very elegant, coherent, and unified understanding of many ideas in nonlinear physics by means of fundamental differential geometric concepts. In fact, relationships between a geometric interpretation of soliton equations, prolongation structure, Lax pairs, and conservation laws can be clearly realized and made use of. The interest in the approach, its generality, and the results it produces do not depend on a specific equation at the outset. The formalism in terms of differential forms [2] can encompass large classes of nonlinear partial differential equation, certainly the AKNS systems [3,4], and it allows the production of generic expressions for infinite numbers of conservation laws. Moreover, it leads to the consequence that many seemingly different equations turn out to be related by a gauge transformation.

Here the discussion begins by studying prolongation structures for a $2 \times 2 S L(2, \mathbb{R})$ system discussed first by Sasaki $[5,6]$ and Crampin [7] to present and illustrate the method. This will also demonstrate the procedure and the kind of prolongation results that emerge. It also provides a basis from which to work out larger systems since they can generally be reduced to $2 \times 2$ problems. Of greater complexity are a pair of $3 \times 3$ problems which will be considered next. It is shown how to construct an $O(3)$ system based on three constituent one forms as well as an $S U(3)$ system composed of eight fundamental one forms. The former has not appeared. The $3 \times$ 3 problem is less well known than the $2 \times 2$ problem; however, some work has appeared in $[8,9]$. In the first of these, the $3 \times 3$ solitons degenerate to the AKNS solitons in three ways, while in the latter nondegenerate case, they do not. All of the results are presented explicitly; that is, the coefficients of all the forms and their higher exterior derivatives are calculated and given explicitly. Maple is used to do this in the harder cases. The approach is quite unified, and so once the formalism is established for the $S L(2, \mathbb{R})$ system, the overall procedure can be carried over to the other Lie algebra cases as well.

Of course, the $3 \times 3$ problems will yield more types of conservation laws. The prolongation structures of the $3 \times 3$ problems will be observed to be reduceable to the same type as the $2 \times 2$ system considered at the beginning. However, the Riccati representations become much more complicated [10]. The generalization of this formalism to an $n \times n$ problem then becomes straightforward after completing the study of these smaller cases [11]. The prolongation structure of an $n \times n$ problem is reduced to a collection of $n$ smaller $(n-1) \times(n-1)$ cases and finally at the end to a set of $2 \times 2$ problems such as the $S L(2, \mathbb{R})$ case at the beginning. Finally, to summarize at 
the end, a collection of conservation laws are developed from one of the results and some speculation as to how this type relates to other procedures $[12,13]$.

\section{2. $S L(2, \mathbb{R})$ Prolongations}

The procedure begins by associating a system of Pfaffian equations to the nonlinear system to be studied, namely,

$$
\alpha_{i}=0, \quad \alpha_{i}=d y_{i}-\Omega_{i j} y_{j}, \quad i, j=1,2 .
$$

In (1), $\Omega$ is a traceless $2 \times 2$ matrix which consists of a family of one forms, $\omega_{i}$. At the end, it is desired to express these one forms in terms of independent variables, which are called $x$ and $t$, the dependent variables and their derivatives. However, no specific equation need be assumed at the start. The underlying structure group is $S L(2, \mathbb{R})$. Explicitly, the matrix of one forms is given by the $2 \times 2$ matrix

$$
\Omega=\left(\begin{array}{cc}
\omega_{1} & \omega_{2} \\
\omega_{3} & -\omega_{1}
\end{array}\right)
$$

The integrability conditions are expressed as the vanishing of a traceless $2 \times 2$ matrix of two forms $\Theta$ given by

$$
\Theta=d \Omega-\Omega \wedge \Omega
$$

In terms of components, the left-hand side of (3) has the form

$$
\Theta=\left(\Theta_{i j}\right)=\left(\begin{array}{cc}
\vartheta_{1} & \vartheta_{2} \\
\vartheta_{3} & -\vartheta_{1}
\end{array}\right) .
$$

Substituting (2) into (3), a matrix which contains the integrability equations in terms of the basis one forms is obtained. The three equations are

$$
\begin{gathered}
\vartheta_{1}=d \omega_{1}-\omega_{2} \wedge \omega_{3}, \quad \vartheta_{2}=d \omega_{2}-2 \omega_{1} \wedge \omega_{2}, \\
\vartheta_{3}=d \omega_{3}+2 \omega_{1} \wedge \omega_{3} .
\end{gathered}
$$

Of use in the theorems which follow, it is also useful to have the $d \omega_{i}$ in (5) expressed explicitly in terms of the $\vartheta_{i}$ as

$$
\begin{gathered}
d \omega_{1}=\vartheta_{1}+\omega_{2} \wedge \omega_{3}, \quad d \omega_{2}=\vartheta_{2}+2 \omega_{1} \wedge \omega_{2}, \\
d \omega_{3}=\vartheta_{3}-2 \omega_{1} \wedge \omega_{3} .
\end{gathered}
$$

Therefore, by selecting a particular choice for the set $\left\{\omega_{i}\right\}$, the nonlinear equation of interest can be written simply as

$$
\Theta=0, \quad \vartheta_{i}=0, \quad i=1,2,3 .
$$

It is easy to see that the system is closed. Upon differentiating $\Theta$ and substituting (3), we obtain

$$
d \Theta=\Omega \wedge \Theta-\Theta \wedge \Omega .
$$

This implies that the exterior derivatives of the set of $\left\{\vartheta_{i}\right\}$ are contained in the ring of two forms $\left\{\vartheta_{i}\right\}$.

Now the differential ideal can be prolonged by including the forms $\left\{\alpha_{1}, \alpha_{2}\right\}$ given by (1).
Theorem 1. The differential one forms

$$
\alpha_{1}=d y_{1}-\omega_{1} y_{1}-\omega_{2} y_{2}, \quad \alpha_{2}=d y_{2}-\omega_{3} y_{1}+\omega_{1} y_{2}
$$

have the following exterior derivatives:

$$
\begin{aligned}
& d \alpha_{1}=\omega_{1} \wedge \alpha_{1}+\omega_{2} \wedge \alpha_{2}-y_{1} \vartheta_{1}-y_{2} \vartheta_{2}, \\
& d \alpha_{2}=-\omega_{1} \wedge \alpha_{2}+\omega_{3} \wedge \alpha_{1}+y_{2} \vartheta_{1}-y_{1} \vartheta_{3} .
\end{aligned}
$$

Hence, the exterior derivatives of the $\left\{\alpha_{i}\right\}$ are contained in the ring of forms $\left\{\vartheta_{i}, \alpha_{j}\right\}$.

Proof. The forms (9) follow by substituting (2) into (1). Differentiating $\alpha_{1}$ in (9) it is found that

$$
d \alpha_{1}=-y_{1} d \omega_{1}+\omega_{1} \wedge d y_{1}-y_{2} d \omega_{2}+\omega_{2} \wedge d y_{2} \text {. }
$$

Obtaining $d y_{1}$ and $d y_{2}$ from (9) and $d \omega_{1}, d \omega_{2}$ from (6), $d \alpha_{1}$ becomes

$$
\begin{aligned}
d \alpha_{1}= & -y_{1} \vartheta_{1}-y_{1} \omega_{2} \wedge \omega_{3}+\omega_{1} \wedge \alpha_{1}+y_{2} \omega_{1} \wedge \omega_{2}-y_{2} \vartheta_{2} \\
& -2 y_{2} \omega_{1} \wedge \omega_{2}+\omega_{2} \wedge \alpha_{2}+y_{1} \omega_{2} \wedge \omega_{3}-y_{2} \omega_{2} \wedge \omega_{1} \\
= & \omega_{1} \wedge \alpha_{1}+\omega_{2} \wedge \alpha_{2}-y_{1} \vartheta_{1}-y_{2} \vartheta_{2} .
\end{aligned}
$$

Similarly, beginning with $\alpha_{2}$,

$$
\begin{aligned}
d \alpha_{2}= & -y_{1} d \omega_{3}+\omega_{3} \wedge d y_{1}+y_{2} d \omega_{1}-\omega_{1} \wedge d y_{2} \\
= & -y_{1} \vartheta_{3}+2 y_{1} \omega_{1} \wedge \omega_{3}+\omega_{3} \wedge \alpha_{1} \\
& +y_{1} \omega_{3} \wedge \omega_{1}+y_{2} \omega_{3} \wedge \omega_{2} \\
& +y_{2} \vartheta_{1}+y_{2} \omega_{2} \wedge \omega_{3}-\omega_{1} \wedge \alpha_{2}-y_{1} \omega_{1} \wedge \omega_{3} \\
= & -\omega_{1} \wedge \alpha_{2}+\omega_{3} \wedge \alpha_{1}+y_{2} \vartheta_{1}-y_{1} \vartheta_{3},
\end{aligned}
$$

as required.

Corollary 2. The exterior derivatives of the $\alpha_{i}$ in (9) can be expressed concisely in terms of the matrix elements of $\Theta$ and $\Omega$ as

$$
d \alpha_{i}=-\Theta_{i j} y_{j}+\Omega_{i j} \wedge \alpha_{j}, \quad i, j=1,2 \text {. }
$$

The corollary is Theorem 1 after using the definitions of the matrices in (2) and (4).

The forms $\alpha_{1}, \alpha_{2}$ given in (9) lead to a natural Riccati representation called $\alpha_{3}, \alpha_{4}$ for this differential system. This arises by taking the following linear combinations of $\alpha_{1}$ and $\alpha_{2}$ :

$$
\begin{aligned}
y_{1}^{2} \alpha_{3} & =y_{1} \alpha_{2}-y_{2} \alpha_{1} \\
& =y_{1} d y_{2}-y_{2} d y_{1}-y_{1}^{2} \omega_{3}+2 y_{1} y_{2} \omega_{1}+y_{2}^{2} \omega_{2}, \\
y_{2}^{2} \alpha_{4} & =y_{2} \alpha_{1}-y_{1} \alpha_{2} \\
& =y_{2} d y_{1}-y_{1} d y_{2}-y_{2}^{2} \omega_{2}-2 y_{1} y_{2} \omega_{1}+y_{1}^{2} \omega_{3} .
\end{aligned}
$$


Two new functions, or pseudopotentials, $y_{3}$ and $y_{4}$ can be introduced which are defined by the transformation

$$
y_{3}=\frac{y_{2}}{y_{1}}, \quad y_{4}=\frac{y_{1}}{y_{2}} .
$$

Then, from (15), the following forms which have a Riccati structure result:

$$
\begin{aligned}
& \alpha_{3}=d y_{3}-\omega_{3}+2 y_{3} \omega_{1}+y_{3}^{2} \omega_{2}, \\
& \alpha_{4}=d y_{4}-\omega_{2}-2 y_{4} \omega_{1}+y_{4}^{2} \omega_{3} .
\end{aligned}
$$

These equations could also be thought of as Riccati equations for $y_{3}$ and $y_{4}$.

Theorem 3. (i) The one forms $\alpha_{3}, \alpha_{4}$ given by (17) have exterior derivatives

$$
\begin{aligned}
& d \alpha_{3}=2 y_{3} \vartheta_{1}+y_{3}^{2} \vartheta_{2}-\vartheta_{3}+2 \alpha_{3} \wedge\left(\omega_{1}+y_{3} \omega_{2}\right), \\
& d \alpha_{4}=-2 y_{4} \vartheta_{1}-\vartheta_{2}+y_{4}^{2} \vartheta_{3}+2 \alpha_{4} \wedge\left(-\omega_{1}+y_{4} \omega_{3}\right) .
\end{aligned}
$$

Consequently, results (18) are contained in the ring spanned by $\left\{\vartheta_{i}\right\}$ and $\left\{\alpha_{j}\right\}$.

(ii) Define the forms $\sigma_{1}=\omega_{1}+y_{3} \omega_{2}$ and $\sigma_{2}=-\omega_{1}+$ $y_{4} \omega_{3}$ appearing in (18). The exterior derivatives of $\sigma_{1}$ and $\sigma_{2}$ are found to be

$$
\begin{aligned}
& d \sigma_{1}=\vartheta_{1}+y_{3} \vartheta_{2}+\alpha_{3} \wedge \omega_{2}, \\
& d \sigma_{2}=-\vartheta_{1}+y_{3} \vartheta_{2}+\alpha_{4} \wedge \omega_{2} .
\end{aligned}
$$

Therefore $d \sigma_{1} \equiv 0$ and $d \sigma_{2} \equiv 0 \bmod \left\{\vartheta_{i}, \alpha_{j}\right\}$.

The proof of this Theorem proceeds along exactly the same lines as Theorem 1 . The $S L(2, \mathbb{R})$ structure and the connection interpretation can be based on the forms $\alpha_{3}, \alpha_{4}$.

At this point, the process can be continued. Two more pseudopotentials $y_{5}$ and $y_{6}$ can be introduced and the differential system can be extended by including two more one forms $\alpha_{5}$ and $\alpha_{6}$. The specific structure of these forms is suggested by Theorem 3 .

Theorem 4. Define two one forms $\alpha_{5}$ and $\alpha_{6}$ as

$$
\alpha_{5}=d y_{5}-\omega_{1}-y_{3} \omega_{2}, \quad \alpha_{6}=d y_{6}+\omega_{1}-y_{4} \omega_{3} .
$$

The exterior derivatives of the one forms (20) are given by the expressions

$$
d \alpha_{5}=-\vartheta_{1}-y_{3} \vartheta_{3}-\alpha_{3} \wedge \omega_{2}, \quad d \alpha_{6}=\vartheta_{1}-y_{4} \vartheta_{3}-\alpha_{4} \wedge \omega_{3} .
$$

Results (21) specify the closure properties of the forms $\alpha_{5}$ and $\alpha_{6}$. This theorem is proved along similar lines keeping in mind that the results for $d y_{3}$ and $d y_{4}$ are obtained from (17).

A final extension can be made by adding two additional pseudopotentials $y_{7}$ and $y_{8}$. At each stage, the ideal of forms is being enlarged and is found to be closed over the underlying subideal which does not contain the two new forms.
Theorem 5. Define the one forms $\alpha_{7}$ and $\alpha_{8}$ by means of

$$
\alpha_{7}=d y_{7}-e^{-2 y_{5}} \omega_{2}, \quad \alpha_{8}=d y_{8}-e^{-2 y_{6}} \omega_{3} .
$$

The exterior derivatives of the forms $\alpha_{7}$ and $\alpha_{8}$ are given by

$$
\begin{aligned}
& d \alpha_{7}=2 e^{-2 y_{5}} \alpha_{5} \wedge \omega_{2}-e^{-2 y_{5}} \vartheta_{2}, \\
& d \alpha_{8}=2 e^{-2 y_{6}} \alpha_{6} \wedge \omega_{3}-e^{-2 y_{6}} \vartheta_{3},
\end{aligned}
$$

and are closed over the prolonged ideal.

\section{3. $O(3)$ Prolongations}

The first $3 \times 3$ problem we consider is formulated in terms of a set of one forms $\omega_{i}$ for $i=1,2,3$. The following system of Pfaffian equations is to be associated to a specific nonlinear equation

$$
\alpha_{i}=0, \quad \alpha_{i}=d y_{i}-\Omega_{i j} y_{j}, \quad i, j=1,2,3 .
$$

In (24), $\Omega$ is a traceless $3 \times 3$ matrix of one forms. The nonlinear equation to emerge is expressed as the vanishing of a traceless $3 \times 3$ matrix of two forms $\Theta$,

$$
\Theta=0, \quad \Theta=d \Omega-\Omega \wedge \Omega .
$$

These can be considered as integrability conditions for (24). The closure property, the gauge transformation, and the gauge theoretic interpretation can also hold for $3 \times 3$ systems. Explicitly the matrix $\Omega$ is given by

$$
\Omega=\left(\begin{array}{ccc}
0 & -\omega_{1} & \omega_{2} \\
\omega_{1} & 0 & -\omega_{3} \\
-\omega_{2} & \omega_{3} & 0
\end{array}\right) .
$$

Using (26) in (24) and (25), the following prolonged differential system is obtained:

$$
\begin{array}{ll}
d y_{1}=\alpha_{1}-y_{2} \omega_{1}+y_{3} \omega_{2}, & d \omega_{1}=\vartheta_{1}-\omega_{2} \wedge \omega_{3}, \\
d y_{2}=\alpha_{2}+y_{1} \omega_{1}-y_{3} \omega_{3}, & d \omega_{2}=\vartheta_{2}-\omega_{3} \wedge \omega_{1}, \\
d y_{3}=\alpha_{3}-y_{1} \omega_{2}+y_{2} \omega_{3}, & d \omega_{3}=\vartheta_{3}-\omega_{1} \wedge \omega_{2} .
\end{array}
$$

By straightforward exterior differentiation of each $\alpha_{i}$ and using (27), the following Theorem results.

Theorem 6. The $\alpha_{i}$ given in (27) have exterior derivatives which are contained in the ring spanned by $\left\{\vartheta_{i}\right\}_{1}^{3}$ and $\left\{\alpha_{j}\right\}_{1}^{3}$ and given explicitly as

$$
\begin{aligned}
& d \alpha_{1}=y_{2} \vartheta_{1}-y_{3} \vartheta_{2}-\omega_{1} \wedge \alpha_{2}+\omega_{2} \wedge \alpha_{3}, \\
& d \alpha_{2}=-y_{1} \vartheta_{1}+y_{3} \vartheta_{3}+\omega_{1} \wedge \alpha_{1}-\omega_{3} \wedge \alpha_{3}, \\
& d \alpha_{3}=y_{1} \vartheta_{2}-y_{2} \vartheta_{3}-\omega_{2} \wedge \alpha_{1}+\omega_{3} \wedge \alpha_{2} .
\end{aligned}
$$

The one forms $\left\{\alpha_{i}\right\}_{1}^{3}$ admit a series of Riccati representations which can be realized by defining six new one forms,

$$
\begin{gathered}
y_{1}^{2} \alpha_{4}=y_{1} \alpha_{2}-y_{2} \alpha_{1}, \quad y_{1}^{2} \alpha_{5}=y_{1} \alpha_{3}-y_{3} \alpha_{1}, \\
y_{2}^{2} \alpha_{6}=y_{2} \alpha_{1}-y_{1} \alpha_{2}, \\
y_{2}^{2} \alpha_{7}=y_{2} \alpha_{3}-y_{3} \alpha_{2}, \quad y_{3}^{2} \alpha_{8}=y_{3} \alpha_{1}-y_{1} \alpha_{3}, \\
y_{3}^{2} \alpha_{9}=y_{3} \alpha_{2}-y_{2} \alpha_{3} .
\end{gathered}
$$


In effect, the larger $3 \times 3$ system is breaking up into several $2 \times 2$ systems. To write the new one forms explicitly, introduce the new functions $\left\{y_{j}\right\}_{4}^{9}$ which are defined in terms of the original $\left\{y_{i}\right\}_{1}^{3}$ as follows:

$$
\begin{aligned}
& y_{4}=\frac{y_{2}}{y_{1}}, \quad y_{5}=\frac{y_{3}}{y_{1}}, \quad y_{6}=\frac{y_{1}}{y_{2}}, \quad y_{7}=\frac{y_{3}}{y_{1}}, \\
& y_{8}=\frac{y_{1}}{y_{3}}, \quad y_{9}=\frac{y_{2}}{y_{3}} \text {. }
\end{aligned}
$$

In terms of the functions defined in (30), the $\left\{\alpha_{j}\right\}_{4}^{9}$ are given as

$$
\begin{aligned}
& \alpha_{4}=d y_{4}-\left(1+y_{4}^{2}\right) \omega_{1}+y_{4} y_{5} \omega_{2}+y_{5} \omega_{3}, \\
& \alpha_{5}=d y_{5}-y_{4} y_{5} \omega_{1}+\left(1+y_{5}^{2}\right) \omega_{2}-y_{4} \omega_{3}, \\
& \alpha_{6}=d y_{6}+\left(1+y_{6}^{2}\right) \omega_{1}-y_{7} \omega_{2}-y_{6} y_{7} \omega_{3}, \\
& \alpha_{7}=d y_{7}+y_{6} y_{7} \omega_{1}+y_{6} \omega_{2}-\left(1+y_{7}^{2}\right) \omega_{3}, \\
& \alpha_{8}=d y_{8}+y_{9} \omega_{1}-\left(1+y_{8}^{2}\right) \omega_{2}+y_{8} y_{9} \omega_{3}, \\
& \alpha_{9}=d y_{9}-y_{8} \omega_{1}-y_{8} y_{9} \omega_{2}+\left(1+y_{9}^{2}\right) \omega_{3} .
\end{aligned}
$$

To state the next theorem, we need to define the following three matrices:

$$
\begin{aligned}
& \Omega_{1}=\left(\begin{array}{cc}
2 y_{4} \omega_{1}-y_{5} \omega_{2} & -y_{4} \omega_{2}-\omega_{3} \\
y_{5} \omega_{1}+\omega_{3} & y_{4} \omega_{1}-2 y_{5} \omega_{2}
\end{array}\right), \\
& \Omega_{2}=\left(\begin{array}{cc}
-2 y_{6} \omega_{1}+y_{7} \omega_{3} & \omega_{2}+y_{6} \omega_{3} \\
-y_{7} \omega_{1}-\omega_{6} & -y_{6} \omega_{1}+2 y_{7} \omega_{3}
\end{array}\right), \\
& \Omega_{3}=\left(\begin{array}{cc}
2 y_{8} \omega_{2}-y_{9} \omega_{3} & -\omega_{1}-y_{9} \omega_{3} \\
\omega_{1}+y_{9} \omega_{2} & y_{8} \omega_{2}-2 y_{9} \omega_{3}
\end{array}\right) .
\end{aligned}
$$

$$
\begin{aligned}
\Theta_{1} & =d \Omega_{1}-\Omega_{1} \wedge \Omega_{1} \\
& =\left(\begin{array}{cc}
2 y_{4} \vartheta_{1}-y_{5} \vartheta_{2}+2 \alpha_{4} \wedge \omega_{1}-\alpha_{5} \wedge \omega_{2} & -y_{4} \vartheta_{2}-\vartheta_{3}-\alpha_{4} \wedge \omega_{2} \\
y_{5} \vartheta_{1}+\vartheta_{3}+\alpha_{5} \wedge \omega_{1} & y_{4} \vartheta_{1}-2 y_{5} \vartheta_{2}+\alpha_{4} \wedge \omega_{1}-2 \alpha_{5} \wedge \omega_{2}
\end{array}\right) .
\end{aligned}
$$

Similar matrix expressions can be found for the cases in which $\Omega_{1}$ is replaced by $\Omega_{2}$ or $\Omega_{3}$, respectively.

Unlike $\Omega$ given in (26), the $\Omega_{i}$ in (32) are not traceless. Define their traces to be

$$
\kappa_{i}=\operatorname{tr} \Omega_{i}, \quad i=1,2,3
$$

These traces provide convenient ways of generating conservation laws on account of the following Theorem.
Theorem 7. The closure properties for the forms $\left\{\alpha_{i}\right\}_{4}^{9}$ given in (31) can be summarized in the form

$$
\begin{aligned}
d\left(\begin{array}{l}
\alpha_{4} \\
\alpha_{5}
\end{array}\right)= & \left(\begin{array}{l}
-\left(1+y_{4}^{2}\right) \vartheta_{1}+y_{4} y_{5} \vartheta_{2}+y_{5} \vartheta_{3} \\
-y_{4} y_{5} \vartheta_{1}+\left(1+y_{5}^{2}\right) \vartheta_{2}-y_{4} \vartheta_{3}
\end{array}\right) \\
& +\Omega_{1} \wedge\left(\begin{array}{c}
\alpha_{4} \\
\alpha_{5}
\end{array}\right), \\
d\left(\begin{array}{c}
\alpha_{6} \\
\alpha_{7}
\end{array}\right)= & \left(\begin{array}{l}
\left(1+y_{6}^{2}\right) \vartheta_{1}-y_{7} \vartheta_{2}-y_{6} y_{7} \vartheta_{3} \\
y_{6} y_{7} \vartheta_{1}+y_{6} \vartheta_{2}-\left(1+y_{7}^{2}\right) \vartheta_{3}
\end{array}\right) \\
& +\Omega_{2} \wedge\left(\begin{array}{c}
\alpha_{6} \\
\alpha_{7}
\end{array}\right), \\
d\left(\begin{array}{c}
\alpha_{8} \\
\alpha_{9}
\end{array}\right)= & \left(\begin{array}{c}
y_{1} \vartheta_{1}-\left(1+y_{8}^{2}\right) \vartheta_{2}+y_{8} y_{9} \vartheta_{3} \\
-y_{8} \vartheta_{1}-y_{8} y_{9} \vartheta_{2}+\left(1+y_{9}^{2}\right) \vartheta_{3}
\end{array}\right) \\
& +\Omega_{3} \wedge\left(\begin{array}{c}
\alpha_{8} \\
\alpha_{9}
\end{array}\right) .
\end{aligned}
$$

The exterior derivatives are therefore contained in the ring spanned by $\left\{\vartheta_{i}\right\}_{1}^{3}$ and the $\left\{\alpha_{j}\right\}_{4}^{9}$.

In terms of $\Omega_{i}$, a corresponding matrix of two forms $\Theta_{i}$ can be defined in terms of the corresponding $\Omega_{i}$ defined in (32),

$$
\Theta_{i}=d \Omega_{i}-\Omega_{i} \wedge \Omega_{i}, \quad i=1,2,3 .
$$

Theorem 8. The $2 \times 2$ matrix of two forms $\Theta_{i}$ defined by (34) is contained in the ring of forms spanned by $\left\{\vartheta_{i}\right\}_{1}^{3}$ coupled with either $\left\{\alpha_{4}, \alpha_{5}\right\},\left\{\alpha_{6}, \alpha_{7}\right\}$, or $\left\{\alpha_{8}, \alpha_{9}\right\}$ when $i=1,2,3$, respectively. Proof. Differentiating $\Omega_{1}$ and simplifying, we have

Theorem 9. The exterior derivatives of traces (36) are given by

$$
\begin{aligned}
& \frac{1}{3} d \kappa_{1}=y_{4} \vartheta_{1}-y_{5} \vartheta_{2}-\omega_{1} \wedge \alpha_{4}+\omega_{2} \wedge \alpha_{5}, \\
& \frac{1}{3} d \kappa_{2}=-y_{6} \vartheta_{1}+y_{7} \vartheta_{3}+\omega_{1} \wedge \alpha_{6}-\omega_{3} \wedge \alpha_{7}, \\
& \frac{1}{3} d \kappa_{3}=y_{8} \vartheta_{2}-y_{9} \vartheta_{3}-\omega_{2} \wedge \alpha_{8}+\omega_{3} \wedge \alpha_{9} .
\end{aligned}
$$

Results (37) are contained in the ring spanned by $\left\{\vartheta_{i}\right\}_{1}^{3}$ and $\left\{\alpha_{j}\right\}_{4}^{9}$. 


\section{4. $S U(3)$ Prolongations}

To formulate a $3 \times 3 S U(3)$ problem, the set of Pfaffian equations,

$$
\alpha_{i}=0, \quad \alpha_{i}=d y_{i}-\Omega_{i j} y_{j}, \quad i, j=1,2,3,
$$

are associated with the nonlinear equation. In (38), $\Omega$ is a traceless $3 \times 3$ matrix consisting of a system of one forms. The nonlinear equation to be considered is expressed as the vanishing of a traceless $3 \times 3$ matrix of two forms $\Theta$ exactly as in (25) which constitute the integrability condition for (38).

A $3 \times 3$ matrix representation of the Lie algebra for $S U(3)$ is introduced by means of generators $\lambda_{j}$ for $j=1, \ldots, 8$, which satisfy the following set of commutation relations:

$$
\left[\lambda_{l}, \lambda_{m}\right]=2 i f_{l m n} \lambda_{n} .
$$

The structure constants $f_{l m n}$ are totally antisymmetric in $l, m, n$. The one form $\Omega$ is expressed in terms of the generators $\lambda_{i}$ as

$$
\Omega=\sum_{l=1}^{8} \omega_{l} \lambda_{l}
$$

Then the two forms $\Theta$ can be written as

$$
\Theta=\sum_{l=1}^{8} \vartheta_{l} \lambda_{l}, \quad \vartheta_{l}=d \omega_{l}-i f_{l m n} \omega_{m} \wedge \omega_{n} .
$$

The nonlinear equation to be solved has the form $\vartheta_{l}=0$ for $l=1, \ldots, 8$.

It will be useful to display (40) and (41) by using the nonzero structure constants given in (39), since these may not be readily accessible. An explicit representation for the matrix $\Omega$ used here is given by

$$
\Omega=\left(\begin{array}{ccc}
\omega_{3}+\frac{1}{\sqrt{3}} \omega_{8} & \omega_{1}-i \omega_{2} & \omega_{4}-i \omega_{5} \\
\omega_{1}+i \omega_{2} & -\omega_{3}+\frac{1}{\sqrt{3}} \omega_{8} & \omega_{6}-i \omega_{7} \\
\omega_{4}+i \omega_{5} & \omega_{6}+i \omega_{7} & -\frac{2}{\sqrt{3}} \omega_{8}
\end{array}\right)
$$

Moreover, in order that the presentation can be easier to follow, the eight forms $d \omega_{i}$ specified by (41) will be given explicitly:

$$
\begin{aligned}
& d \omega_{1}=\vartheta_{1}+2 i \omega_{2} \wedge \omega_{3}+i \omega_{4} \wedge \omega_{7}-i \omega_{5} \wedge \omega_{6} \\
& d \omega_{2}=\vartheta_{2}-2 i \omega_{1} \wedge \omega_{3}+i \omega_{4} \wedge \omega_{6}+i \omega_{5} \wedge \omega_{7} \\
& d \omega_{3}=\vartheta_{3}+2 i \omega_{1} \wedge \omega_{2}+i \omega_{4} \wedge \omega_{5}-i \omega_{6} \wedge \omega_{7} \\
& d \omega_{4}=\vartheta_{4}-i \omega_{1} \wedge \omega_{7}-i \omega_{2} \wedge \omega_{6}-i \omega_{3} \wedge \omega_{5}+\sqrt{3} i \omega_{5} \wedge \omega_{8} \\
& d \omega_{5}=\vartheta_{5}+i \omega_{1} \wedge \omega_{6}-i \omega_{2} \wedge \omega_{7}+i \omega_{3} \wedge \omega_{4}-\sqrt{3} i \omega_{4} \wedge \omega_{8}, \\
& d \omega_{6}=\vartheta_{6}-i \omega_{1} \wedge \omega_{5}+i \omega_{2} \wedge \omega_{4}+i \omega_{3} \wedge \omega_{7}+\sqrt{3} i \omega_{7} \wedge \omega_{8}, \\
& d \omega_{7}=\vartheta_{7}+i \omega_{1} \wedge \omega_{4}+i \omega_{2} \wedge \omega_{5}-i \omega_{3} \wedge \omega_{6}-\sqrt{3} i \omega_{6} \wedge \omega_{8} \\
& d \omega_{8}=\vartheta_{8}+\sqrt{3} i \omega_{4} \wedge \omega_{5}+\sqrt{3} i \omega_{6} \wedge \omega_{7} .
\end{aligned}
$$

Substituting (42) into (38), the following system of one forms $\left\{\alpha_{i}\right\}_{1}^{3}$ is obtained,

$$
\begin{aligned}
& \alpha_{1}=d y_{1}-\left(\omega_{3}+\frac{1}{\sqrt{3}} \omega_{8}\right) y_{1}-\left(\omega_{1}-i \omega_{2}\right) y_{2}-\left(\omega_{4}-i \omega_{5}\right) y_{3}, \\
& \alpha_{2}=d y_{2}-\left(\omega_{1}+i \omega_{2}\right) y_{1}+\left(\omega_{3}-\frac{1}{\sqrt{3}} \omega_{8}\right) y_{2}-\left(\omega_{6}-i \omega_{7}\right) y_{3}, \\
& \alpha_{3}=d y_{3}-\left(\omega_{4}+i \omega_{5}\right) y_{1}-\left(\omega_{6}+i \omega_{7}\right) y_{2}+\frac{2}{\sqrt{3}} \omega_{8} y_{3} .
\end{aligned}
$$

The increase in complexity of this system makes it often necessary to resort to the use of symbolic manipulation to carry out longer calculations, and for the most part, only results are given.

Theorem 10. The exterior derivatives of the $\alpha_{i}$ in (44) are given by

$$
\begin{aligned}
d \alpha_{1}= & -y_{2}\left(\vartheta_{1}-i \vartheta_{2}\right)-y_{1} \vartheta_{3}-y_{3}\left(\vartheta_{4}-i \vartheta_{5}\right) \\
& -\frac{1}{\sqrt{3}} y_{1} \vartheta_{8}+\left(\omega_{3}+\frac{1}{\sqrt{3}} \omega_{8}\right) \wedge \alpha_{1} \\
& +\left(\omega_{1}-i \omega_{2}\right) \wedge \alpha_{2}+\left(\omega_{4}-i \omega_{5}\right) \wedge \alpha_{3}, \\
d \alpha_{2}= & -y_{1}\left(\vartheta_{1}+i \vartheta_{2}\right)+y_{2} \vartheta_{3} \\
& -\frac{1}{\sqrt{3}} y_{2} \vartheta_{8}-y_{3}\left(\vartheta_{6}-i \vartheta_{7}\right)+\left(\omega_{1}+i \omega_{2}\right) \wedge \alpha_{1} \\
& -\left(\omega_{3}-\frac{1}{\sqrt{3}} \omega_{8}\right) \wedge \alpha_{2}+\left(\omega_{6}-i \omega_{7}\right) \wedge \alpha_{3}, \\
d \alpha_{3}= & -y_{1}\left(\vartheta_{4}+i \vartheta_{5}\right)-y_{2}\left(\vartheta_{6}+i \vartheta_{7}\right) \\
& +\frac{2}{\sqrt{3}} y_{3} \vartheta_{8}+\left(\omega_{4}+i \omega_{5}\right) \wedge \alpha_{1} \\
& +\left(\omega_{6}+i \omega_{7}\right) \wedge \alpha_{2}-\frac{2}{\sqrt{3}} \omega_{8} \wedge \alpha_{3} .
\end{aligned}
$$

In order to keep the notation concise, an abbreviation will be introduced. Suppose that $\left\{\gamma_{i}\right\}$ is a system of forms, and $i, j$ are integers, then we make the abbreviation

$$
\gamma_{i j \pm}=\gamma_{i} \pm i \gamma_{j}
$$

At this point, quadratic pseudopotentials can be introduced in terms of the homogeneous variables of the same form as 
(30). In the same way that (31) was produced, the following Pfaffian equations based on the set $\left\{\alpha_{i}\right\}_{1}^{3}$ in (44) are obtained:

$$
\begin{aligned}
\alpha_{4}= & d y_{4}-\omega_{12+}+2 y_{4} \omega_{3}+y_{4}^{2} \omega_{12-} \\
& -y_{5} \omega_{67-}+y_{4} y_{5} \omega_{45-}, \\
\alpha_{5}= & d y_{5}-\omega_{45+}+y_{5}\left(\omega_{3}+\sqrt{3} \omega_{8}\right) \\
& +y_{5}^{2} \omega_{45-}-y_{4} \omega_{67+}+y_{4} y_{5} \omega_{12-}, \\
\alpha_{6}= & d y_{6}-\omega_{12-}-2 y_{6} \omega_{3}+y_{6}^{2} \omega_{12+} \\
& -y_{7} \omega_{45-}+y_{6} y_{7} \omega_{67-}, \\
\alpha_{7}= & d y_{7}-\omega_{67+}-y_{7}\left(\omega_{3}-\sqrt{3} \omega_{8}\right) \\
& +y_{7}^{2} \omega_{67-}-y_{6} \omega_{45+}+y_{6} y_{7} \omega_{12+}, \\
\alpha_{8}= & d y_{8}-\omega_{45-}-y_{8}\left(\omega_{3}+\sqrt{3} \omega_{8}\right) \\
& +y_{8}^{2} \omega_{45+}-y_{9} \omega_{12-}+y_{8} y_{9} \omega_{67+}, \\
\alpha_{9}= & d y_{9}-\omega_{67-}+y_{9}\left(\omega_{3}-\sqrt{3} \omega_{8}\right) \\
& +y_{9}^{2} \omega_{67+}-y_{8} \omega_{12+}+y_{8} y_{9} \omega_{45+} \cdot
\end{aligned}
$$

These are coupled Riccati equations for the pairs of pseudopotentials $\left(y_{4}, y_{5}\right),\left(y_{6}, y_{7}\right)$, and $\left(y_{8}, y_{9}\right)$, respectively.

Define the following set of $2 \times 2$ matrices of one forms $\Omega_{i}$ for $i=1,2,3$ as

$$
\begin{gathered}
\Omega_{1}=\left(\begin{array}{cc}
-2 \omega_{3}-2 y_{4} \omega_{12-}-y_{5} \omega_{45-} & \omega_{67-}-y_{4} \omega_{45-} \\
\omega_{67+}-y_{5} \omega_{12-} & -\omega_{3}-\sqrt{3} \omega_{8}-y_{4} \omega_{12-}-2 y_{5} \omega_{45-}
\end{array}\right), \\
\Omega_{2}=\left(\begin{array}{cc}
2 \omega_{3}-2 y_{6} \omega_{12+}-y_{7} \omega_{67-} & \omega_{45-}-y_{6} \omega_{67-} \\
\omega_{45+}-y_{7} \omega_{12+} & \omega_{3}-\sqrt{3} \omega_{8}-y_{6} \omega_{12+}-2 y_{7} \omega_{67-}
\end{array}\right), \\
\Omega_{3}=\left(\begin{array}{cc}
\omega_{3}+\sqrt{3} \omega_{8}-2 y_{8} \omega_{45+}-y_{9} \omega_{67+} & \omega_{12-}-y_{8} \omega_{67+} \\
\omega_{12+}-y_{9} \omega_{45+} & -\omega_{3}+\sqrt{3} \omega_{8}-y_{8} \omega_{45+}-2 y_{9} \omega_{67+}
\end{array}\right) .
\end{gathered}
$$

Making use of the matrices $\Omega_{i}$ defined in (48), the following result can be stated.

Theorem 11. The closure properties of the set of forms $\left\{\alpha_{i}\right\}_{4}^{9}$ given by (47) can be expressed in terms of the $\Omega_{i}$ as follows:

$$
\begin{aligned}
& d\left(\begin{array}{l}
\alpha_{4} \\
\alpha_{5}
\end{array}\right) \\
& =\left(\begin{array}{c}
\left(y_{4}^{2}-1\right) \vartheta_{1}-i\left(y_{4}^{2}+1\right) \vartheta_{2}+2 y_{4} \vartheta_{3}+y_{4} y_{5} \vartheta_{45-}-y_{5} \vartheta_{67-} \\
y_{4} y_{5} \vartheta_{12-}+y_{5} \vartheta_{3}+\left(y_{4}^{2}-1\right) \vartheta_{4}-i\left(y_{5}^{2}+1\right) \vartheta_{5}-y_{4} \vartheta_{67+}+\sqrt{3} y_{5} \vartheta_{8}
\end{array}\right) \\
& \quad+\Omega_{1} \wedge\left(\begin{array}{c}
\alpha_{4} \\
\alpha_{5}
\end{array}\right), \\
& d\left(\begin{array}{c}
\alpha_{6} \\
\alpha_{7}
\end{array}\right) \\
& =\left(\begin{array}{c}
\left(y_{6}^{2}-1\right) \vartheta_{1}+i\left(y_{6}^{2}+1\right) \vartheta_{2}-2 y_{6} \vartheta_{3}-y_{7} \vartheta_{45-}+y_{6} y_{7} \vartheta_{67-} \\
y_{6} y_{7} \vartheta_{12+}-y_{7} \vartheta_{3}-y_{6} \vartheta_{45+}+\left(y_{7}^{2}-1\right) \vartheta_{6}-i\left(y_{7}^{2}+1\right) \vartheta_{7}+\sqrt{3} y_{7} \vartheta_{8}
\end{array}\right) \\
& \quad+\Omega_{2} \wedge\left(\begin{array}{c}
\alpha_{6} \\
\alpha_{7}
\end{array}\right),
\end{aligned}
$$

$$
\begin{aligned}
& d\left(\begin{array}{l}
\alpha_{8} \\
\alpha_{9}
\end{array}\right) \\
& =\left(\begin{array}{l}
-y_{9} \vartheta_{12-}-y_{8} \vartheta_{3}+\left(y_{8}^{2}-1\right) \vartheta_{4}+i\left(y_{8}^{2}+1\right) \vartheta_{5}+y_{8} y_{9} \vartheta_{67+}-\sqrt{3} y_{8} \vartheta_{8} \\
-y_{8} \vartheta_{12+}+y_{9} \vartheta_{3}+y_{8} y_{9} \vartheta_{45+}+\left(y_{9}^{2}-1\right) \vartheta_{6}+i\left(y_{9}^{2}+1\right) \vartheta_{7}-\sqrt{3} y_{9} \vartheta_{8}
\end{array}\right) \\
& +\Omega_{3} \wedge\left(\begin{array}{c}
\alpha_{8} \\
\alpha_{9}
\end{array}\right) .
\end{aligned}
$$

Based on the forms $\Omega_{i}$ given in (48), we can differentiate to define the following matrices of two forms $\xi_{i}$ given by

$$
\xi_{i}=d \Omega_{i}-\Omega_{i} \wedge \Omega_{i}
$$

Theorem 12. The forms $\xi_{i}$ defined in (50) are given explicitly by the matrices

$\xi_{1}$

$$
=\left(\begin{array}{cc}
2 \omega_{12+} \wedge \alpha_{4}+\omega_{45-} \wedge \alpha_{5} & \omega_{45-} \wedge \alpha_{4}-y_{4} \vartheta_{45-}+\vartheta_{67-} \\
-2 y_{4} \vartheta_{12-}-2 \vartheta_{3}-y_{5} \vartheta_{45-} & \omega_{12-} \wedge \alpha_{4}+2 \omega_{45-} \wedge \alpha_{5} \\
\omega_{12-} \wedge \alpha_{5}-y_{5} \vartheta_{12-}+\vartheta_{67+} & -y_{4} \vartheta_{12-}-\vartheta_{3}-2 \vartheta_{45-}-\sqrt{3} \vartheta_{8}
\end{array}\right),
$$

$\xi_{2}$

$$
\begin{aligned}
= & \left(\begin{array}{cc}
2 \omega_{12+} \wedge \alpha_{6}+\omega_{67-} \wedge \alpha_{7} & \omega_{67-} \wedge \alpha_{6}+\vartheta_{45-}-y_{6} \vartheta_{67-} \\
-2 y_{6} \vartheta_{12+}+\vartheta_{3}-y_{7} \vartheta_{67-} & \omega_{12+} \wedge \alpha_{6}+2 \omega_{67-} \wedge \alpha_{7}-y_{6} \vartheta_{12+} \\
\omega_{12+} \wedge \alpha_{7}-y_{7} \vartheta_{12+}+\vartheta_{45+} & +\vartheta_{3}-2 y_{7} \vartheta_{67-}-\sqrt{3} \vartheta_{8}
\end{array}\right), \\
& \xi_{3} \\
= & \left(\begin{array}{cc}
2 \omega_{45+} \wedge \alpha_{8}+\omega_{67+} \wedge \alpha_{9}+\vartheta_{3} & \omega_{67+} \wedge \alpha_{8}+\vartheta_{12-}-y_{8} \vartheta_{67+} \\
-2 y_{8} \vartheta_{45+}-y_{9} \vartheta_{67+}+\sqrt{3} \vartheta_{8} & \omega_{45+} \wedge \alpha_{8}+2 \omega_{67+} \wedge \alpha_{9}-\vartheta_{3} \\
\omega_{45+} \wedge \alpha_{9}+\vartheta_{12+}-y_{9} \vartheta_{45+} & -y_{8} \vartheta_{45+}-2 y_{9} \vartheta_{67+}+\sqrt{3} \vartheta_{8}
\end{array}\right) .
\end{aligned}
$$

From these results, it is concluded that $\xi_{1}$ is contained in the ring of $\left\{\alpha_{4}, \alpha_{5}\right\}$ and the $\left\{\vartheta_{l}\right\}, \xi_{2}$ is contained in the ring of $\left\{\alpha_{6}, \alpha_{7}\right\}$ and $\left\{\vartheta_{l}\right\}$, and $\xi_{3}$ is in the ring of $\left\{\alpha_{8}, \alpha_{9}\right\}$ and $\left\{\vartheta_{l}\right\}$.

The two forms $\left\{\xi_{i}\right\}_{1}^{3}$ therefore vanish for the solutions of the nonlinear equations (39) and of the coupled Riccati equations $\left\{\alpha_{j}=0\right\}_{4}^{9}$. As in the case of $\mathrm{O}(3)$, the one forms $\Omega_{i}$ are not traceless. Denoting the trace of $\Omega_{i}$ by

$$
\tau_{i}=\operatorname{tr} \Omega_{i}
$$

the following theorem then follows.

Theorem 13. The closure properties of the exterior derivatives of the traces $\tau_{i}$ are given by

$$
\begin{aligned}
\frac{1}{3} d \tau_{1}= & \omega_{12-} \wedge \alpha_{4}+\omega_{45-} \wedge \alpha_{5} \\
& -y_{4} \vartheta_{12-}-\vartheta_{3}-y_{5} \vartheta_{45-}-\frac{1}{\sqrt{3}} \vartheta_{8},
\end{aligned}
$$




$$
\begin{aligned}
\frac{1}{3} d \tau_{2}= & \omega_{12+} \wedge \alpha_{6}+\omega_{67-} \wedge \alpha_{7} \\
& -y_{6} \vartheta_{12+}+\vartheta_{3}-y_{7} \vartheta_{67-}-\frac{1}{\sqrt{3}} \vartheta_{8} \\
\frac{1}{3} d \tau_{3}= & \omega_{45+} \wedge \alpha_{8}+\omega_{67+} \wedge \alpha_{9} \\
& -y_{8} \vartheta_{45+}-y_{9} \vartheta_{67+}+\frac{2}{\sqrt{3}} \vartheta_{8}
\end{aligned}
$$

The one forms (52) then can be used to generate conservation laws. Note that as in the $O(3)$ case, a $3 \times 3$ problem $\Omega$ has been reduced to three separate $2 \times 2$ problems in terms of the matrices $\Omega_{l}$. Further prolongation can be continued beginning with the one forms $\Omega_{l}$ given in (48). To briefly outline the further steps in the procedure, a system of Pfaffians $\left\{\widetilde{\alpha}_{l j}\right\}$ is introduced which are based on the one forms $\Omega_{l}$

$$
\widetilde{\alpha}_{l j}=0, \quad \widetilde{\alpha}_{l j}=d y_{l j}-\left(\Omega_{l}\right)_{j k} y_{l k} .
$$

The subscript $l$ in $y_{l j}$ and $\widetilde{\alpha}_{l j}$ indicates that they belong to the subsystem defined by $\Omega_{l}$, and it is not summed over. Exterior differentiation of $\widetilde{\alpha}_{l j}$ yields

$$
d \widetilde{\alpha}_{l j}=\left(\Omega_{l}\right)_{j k} \wedge \widetilde{\alpha}_{l k}-(\widetilde{\Theta})_{j k} y_{l k}
$$

The $\left\{y_{l j}\right\}$ are pseudopotentials for the original nonlinear equation (39). Next, quadratic pseudopotentials are included as the homogeneous variables and the prolongation can be continued.

\section{A Conservation Law and Summary}

These types of results turn out to be very useful for further study of integrable equations. They can be used for generating infinite numbers of conservation laws. In addition, the results are independent of any further structure of the forms $\omega_{i}$ in all cases. In fact, the one form $\Omega$ need not be thought of as unique. This is due to the fact that $\Omega$ and $\Theta$ are form invariant under the gauge transformation $\Omega \rightarrow \omega^{\prime}=d A A^{-1}+A \Omega A^{-1}$ and $\Theta \rightarrow \Theta^{\prime}=A \Theta A^{-1}$ where, in the $S L(2, \mathbb{R})$ case, $A$ is an arbitrary $2 \times 2$ space-time-dependent matrix of determinant one. The gauge transformation property holds in the $3 \times 3$ case as well. The pseudopotentials serve as potentials for conservation laws in a generalized sense. They can be defined under a choice of Pfaffian forms such as

$$
\alpha_{i}=0, \quad \alpha_{i}=d y_{i}+F_{i} d x+G_{i} d t,
$$

with the property that the exterior derivatives $d \alpha_{i}$ are contained in the ring spanned by $\left\{\alpha_{i}\right\}$ and $\left\{\vartheta_{l}\right\}$

$$
d \alpha_{i}=\sum_{j} A_{i j} \wedge \alpha_{j}+\sum_{l} \Gamma_{i l} \vartheta_{l} .
$$

This can be thought of as a generalization of the Frobenius Theorem for complete integrability of Pfaffian systems.
Theorem 3 implies that $d \sigma_{i}=0$ when $i=1,2$ for solutions of the original equation. If either of these forms is expressed as $\sigma=\mathscr{I} d x+\mathscr{J} d t$, then $d \sigma=0$ implies that

$$
\frac{\partial \mathscr{I}}{\partial t}-\frac{\partial \mathscr{J}}{\partial t}=0
$$

Thus $\mathscr{I}$ is a conserved density and $\mathscr{J}$ a conserved current. Let us finally represent $\omega_{1}=a_{1} d x+b_{1} d t, \omega_{2}=a_{2} d x+b_{2} d t$, and $\omega_{3}=\eta d x+b_{3} d t$, where $\eta$ is a parameter. The $x$-dependent piece of $\alpha_{3}$ in (17) implies

$$
y_{3, x}+2 a_{1} y_{3}+a_{2} y_{3}^{2}-\eta=0
$$

By substituting an asymptotic expansion around $\eta=\infty$ for $y_{3}$ in (59) of the form,

$$
y_{3}=\sum_{0}^{\infty} \eta^{-n} Y_{n}
$$

a recursion for the $Y_{n}$ is obtained. Thus (59) becomes,

$$
\begin{aligned}
& \sum_{n} \eta^{-n} Y_{n, x}+2 a_{1} \sum_{n} \eta^{-n} Y_{n} \\
& \quad+a_{2}\left(\sum_{n} \eta^{-n} Y_{n}\right)^{2}-\eta=0 .
\end{aligned}
$$

Expanding the power, collecting coefficients of $\eta$, and equating coefficients of $\eta$ to zero, we get

$$
Y_{1, x}+2 a_{1} Y_{1}=1, \quad Y_{n, x}+2 a_{1} Y_{n}+a_{2} \sum_{k=1}^{n-1} Y_{n-k} Y_{k}=0
$$

The consistency of solving $\alpha_{3}$ by using just the $x$ part is guaranteed by complete integrability. The $x$ part of the form $\sigma_{1}$ is expressed as

$$
\left(\sigma_{1}\right)_{x}=\left(a_{1}+\sum_{n=1}^{\infty} \eta^{-n} Y_{n} a_{2}\right) d x
$$

Consequently, from (58) the $n$th conserved density is

$$
\mathscr{I}_{n}=a_{2} Y_{n}
$$

The existence of links between the types of prolongation here and other types of prolongations which are based on closed differential systems is a subject for further work.

\section{References}

[1] F. B. Estabrook and H. D. Wahlquist, "Classical geometries defined by exterior differential systems on higher frame bundles," Classical and Quantum Gravity, vol. 6, no. 3, pp. 263-274, 1989.

[2] H. Cartan, Differential Forms, Dover, Mineola, NY, USA, 2006.

[3] M. J. Ablowitz, D. J. Kaup, A. C. Newell, and H. Segur, "Method for solving the sine-Gordon equation," Physical Review Letters, vol. 30, pp. 1262-1264, 1973. 
[4] M. J. Ablowitz, D. K. Kaup, A. C. Newell, and H. Segur, "Nonlinear-evolution equations of physical significance," Physical Review Letters, vol. 31, pp. 125-127, 1973.

[5] R. Sasaki, "Pseudopotentials for the general AKNS system," Physics Letters A, vol. 73, no. 2, pp. 77-80, 1979.

[6] R. Sasaki, "Geometric approach to soliton equations," Proceedings of the Royal Society. London, vol. 373, no. 1754, pp. 373-384, 1980.

[7] M. Crampin, "Solitons and $S L(2, \mathbb{R})$," Physics Letters A, vol. 66, no. 3, pp. 170-172, 1978.

[8] D. J. Kaup and J. Yang, "The inverse scattering transform and squared eigenfunctions for a degenerate $3 \times 3$ operator," Inverse Problems, vol. 25, no. 10, Article ID 105010, 2009.

[9] D. J. Kaup and R. A. Van Gorder, "The inverse scattering transform and squared eigenfunctions for the nondegenerate $3 \times 3$ operator and its soliton structure," Inverse Problems, vol. 26, no. 5, Article ID 055005, 2010.

[10] P. Bracken, "Intrinsic formulation of geometric integrability and associated Riccati system generating conservation laws," International Journal of Geometric Methods in Modern Physics, vol. 6, no. 5, pp. 825-837, 2009.

[11] D. J. Kaup and R. A. Van Gorder, "Squared eigenfunctions and the perturbation theory for the nondegenerate $\mathrm{N} \times \mathrm{N}$ operator: a general outline," Journal of Physics A, vol. 43, Article ID 434019, 2010.

[12] H. D. Wahlquist and F. B. Estabrook, "Prolongation structures of nonlinear evolution equations," Journal of Mathematical Physics, vol. 16, pp. 1-7, 1975.

[13] P. Bracken, "On two-dimensional manifolds with constant Gaussian curvature and their associated equations," International Journal of Geometric Methods in Modern Physics, vol. 9, no. 3, Article ID 1250018, 2012. 


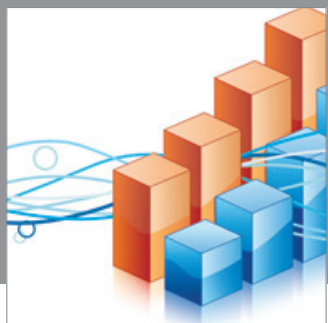

Advances in

Operations Research

mansans

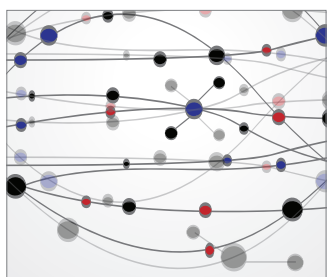

The Scientific World Journal
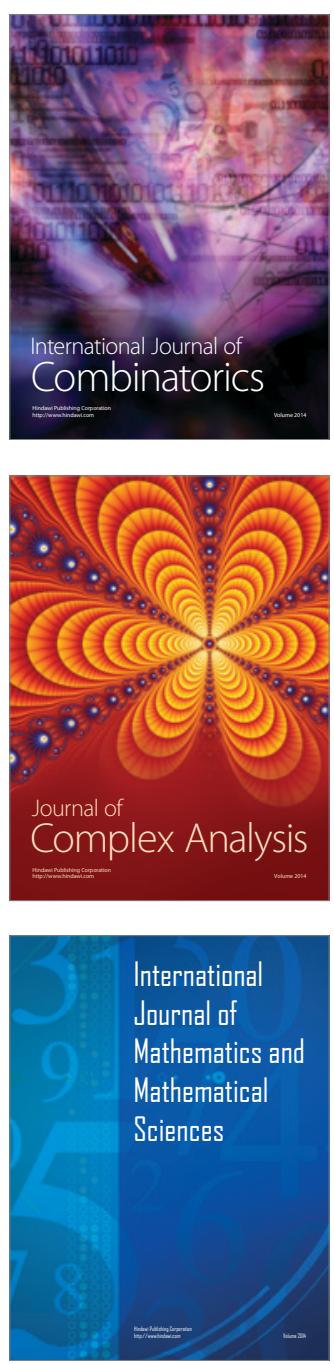
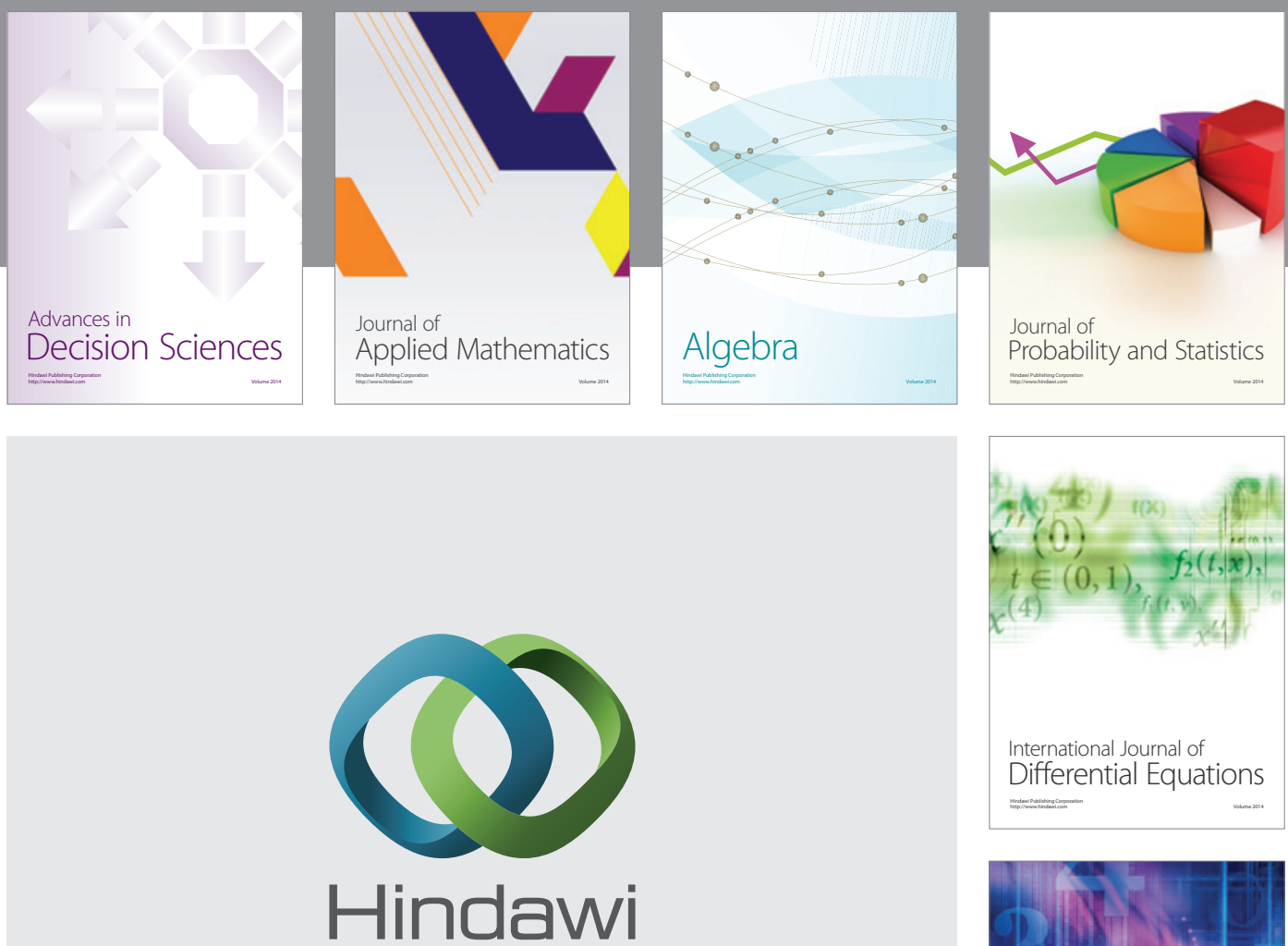

Submit your manuscripts at http://www.hindawi.com
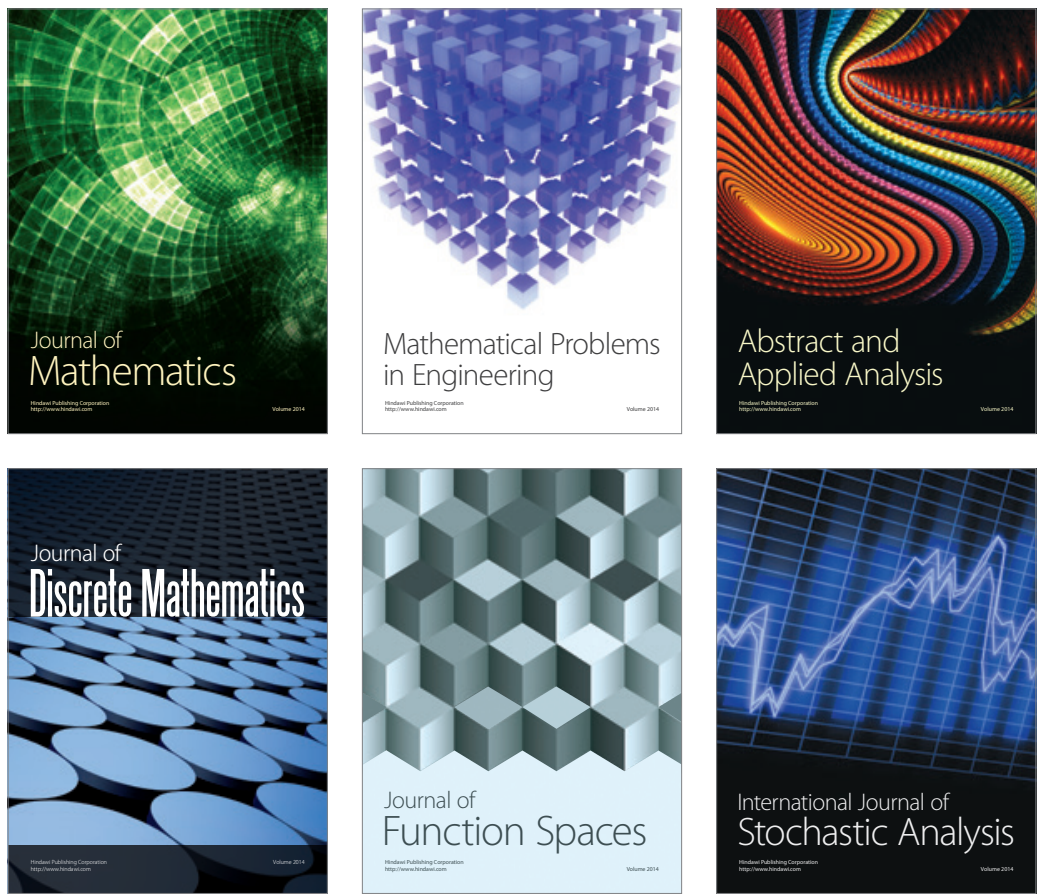

Journal of

Function Spaces

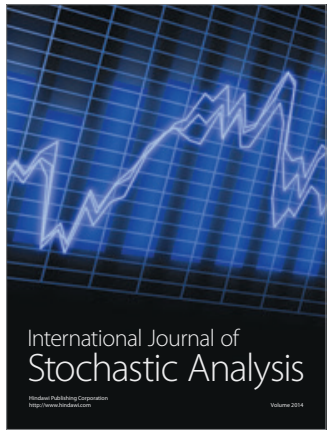

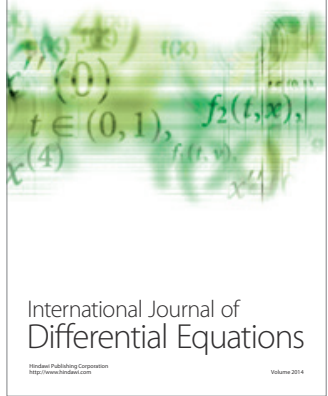
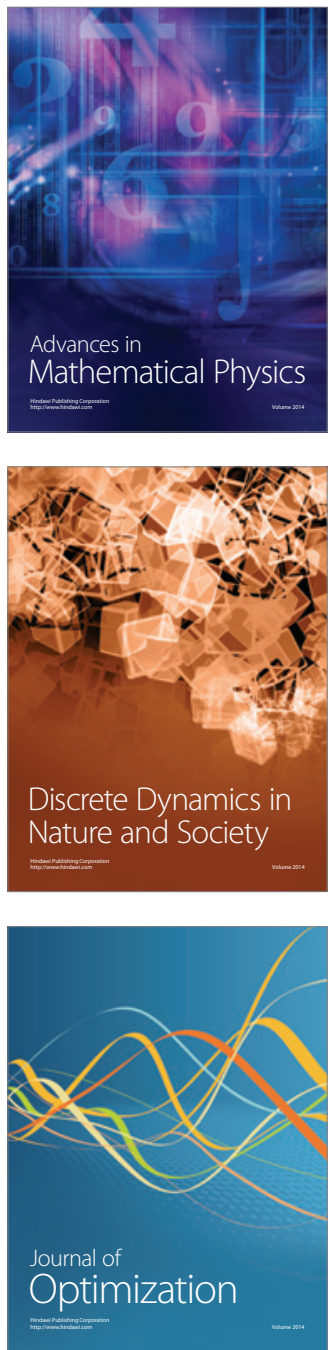